Ann. Geophysicae 16, 812-820 (1998) @ C EGS - Springer-Verlag 1998

\title{
Spatial structure of auroral day-time ionospheric electron density irregularities generated by a powerful HF-wave
}

\author{
E. D. Tereshchenko ${ }^{1}$, B. Z. Khudukon ${ }^{1}$, M. T. Rietveld ${ }^{2,3}$, A. Brekke ${ }^{4}$ \\ ${ }^{1}$ Polar Geophysical Institute, 15 Khalturina St. 183010 Murmansk, Russia \\ ${ }^{2}$ EISCAT, N-9020 Tromsdalen, Norway \\ ${ }^{3}$ Also at Max-Planck-Institut für Aeronomie, D-37191 Katlenburg-Lindau, Germany \\ ${ }^{4}$ University of Tromsø, N-9037 Tromsø, Norway
}

Received: 30 May 1997/ Revised: 3 February 1998 / Accepted: 10 February 1998

\begin{abstract}
We describe an experiment in satellite radiowave probing of the ionosphere, modified by powerful waves from the HF heating facility at Tromsø (Norway) in May 1995. Amplitude scintillations and variations of the phase of VHF signals from Russian navigational satellites passing over the heated region were observed. We show that both large-scale electron density irregularities (several tens of kilometers in size) and small-scale ones (from hundreds of meters to kilometers) can be generated by the HF radiation. Maximum effects caused by small-scale irregularities detected in the satellite signals are observed in the directions sector approximately parallel to the geomagnetic field lines although large-scale structures can be detected within a much larger area. The properties of small-scale irregularities (electron density fluctuations) are investigated by applying a statistical analysis and by studying experimental and model mean values of the logarithm of the relative amplitude of the signal. The results indicate that satellite radio probing can be a supporting diagnostic technique for ionospheric heating and add valuable information to studies of effects produced by $\mathrm{HF}$ modification.
\end{abstract}

Key words. Satellite radio-wave probing $\cdot \mathrm{HF}$ radiation $\cdot$ Electron density irregularities $\cdot$ Statistical analysis $\cdot$ Ionospheric heating

\section{Introduction}

Heating experiments dedicated to studies of ionospheric modification by powerful HF-radiation have a considerable history. The physics of the heating problems can

Correspondence to: E. D. Tereschenko be found in the research by Ginsburg and Gurevich (1960), Farley (1963), and Gurevich (1978) which provided the impetus for experimental programmes using the facilities at Platteville (Colorado, USA), Arecibo (Puerto Rico), Nizhny Novgorod (former Gorky, Russia), Kola Peninsula (Russia) and Tromsø (Norway). One of the physical effects of HF ionospheric modification is the generation of electron density irregularities resulting in an artificially spread F-layer seen on ionograms, scintillation of signals from radio stars and satellites as well as changes in signals scattered from the heated region, in particular, in the spectra of incoherent scatter signals. The studies of heating effects and experimental results can be found in many papers reviewed by Stubbe (1996). The study of artificial largescale irregularities is described by Basu et al. (1987) and more recent studies using the Tromsø heating facility were made by Costa et al. (1997). The observations made show that electron density irregularities with scales ranging from tens of meters to kilometers can be produced in the ionosphere. It was suggested by Gurevich (1978) and Perkins and Valeo (1974) that the self-focusing instability is the main mechanism for producing the irregularities, as a result of focusing and defocusing of the heating wave, in the region of small density perturbations caused by the wave. Therefore, the characteristics of background fields, in particular the magnetic field of the high-latitude ionosphere, as well as the condition of the background ionosphere, are expected to play an important role in the process of irregularity generation. A distinctive property of the polar ionosphere is that the magnetic field lines are oriented almost vertically promoting an easy plasma transport along the field lines and, as a result, the formation of elongated vertical structures. The existence of natural irregularities in the electron density also changes the generation process of irregularities. It is therefore necessary to survey the background conditions in the ionosphere in order to distinguish between natural and artificial density inhomogeneities when performing heating experiments. 
We consider the electron density modification by the powerful radiation of the HF heating facility at Tromsø during a short experiment in May 1995. In earlier papers e.g. by Basu et al. (1987) stationary or quasi-stationary radio sources were used to observe heated regions. This work presents results of ionospheric probing by signals from fast-moving satellites on almost polar orbits carrying differential Doppler experiments. Both amplitude scintillations and phase variations are studied allowing us to estimate the boundaries of the modified region. Information about density irregularities within a wide spectrum of spatial scales ranging from hundreds of meters to tens of kilometers can be obtained. In the following section the experimental arrangement and the receiving equipment used for measuring the amplitude and phase of satellite signals are described. The relevant theoretical background is then presented and the experimental data are discussed. Finally we summarise the results obtained.

\section{Description of the experiment}

The ionospheric modification experiment was carried out by powerful HF waves at Ramfjordmoen near Tromsø $\left(69.6^{\circ} \mathrm{N}, 19.2^{\circ} \mathrm{E}, \mathrm{L}=6.2\right.$, magnetic dip angle $\mathrm{I}=78^{\circ}$ ) simultaneously with the measurements of amplitude and phase of signals from navigational satellites made at the same location on 17-20 May, 1995. The technical description of the facility and its parameters can be found in Rietveld et al. (1993) and here we note only some details related to the measurements. The facility can generate up to $1.2 \mathrm{MW}$ of $\mathrm{CW}$ power in the frequency range from 3.85 to $8 \mathrm{MHz}$. The antenna system consists of 3 antenna arrays designed to cover this frequency range. Array 2 was used since at that time the ionosonde maximum critical frequency in the F-region varied from 4 to $5 \mathrm{MHz}$. Array 2 contains 6 rows of 6 crossed dipoles ( 270 by $270 \mathrm{~m}$ ) to cover the range 3.85-5.65 MHz. During the experiment the antenna beam was pointed vertically and a $4.04 \mathrm{MHz}-$ wave was transmitted. The radiation wave power in
$\mathrm{CW}$-mode was $12 \times 90 \mathrm{~kW}=1080 \mathrm{~kW}$. This corresponds to $270 \mathrm{MW}$ of effective radiated power in all the experiments. Russian navigational satellites were used to measure the amplitude and phase of the signals propagating through the heated region. The satellite system is described by Wood and Perry (1980). The nearly polar-orbiting navigational satellites at a height of about $1000 \mathrm{~km}$ have an orbit inclination of $83^{\circ}$, therefore the trajectory projection on the ground for satellites moving from the north lies approximately along the geomagnetic meridian. The beamwidth of array 2 is about $15^{\circ}$ so it takes less than $40 \mathrm{~s}$ for a satellite to cross the beam allowing for observations of temporal changes of the signal caused by a spatial inhomogeneity of the medium. Only passes of satellites with such a high elevation that they could cross the ionospheric region, illuminated by the main lobe of the heating facility, have been recorded. In total five records have been made during the heating experiment. All the relevant experimental parameters are given in Table 1.

The satellite records for experiments 3 and 4 do not show any visible changes due to heating effects either in the amplitude or in the phase. Also, the ionograms do not show any ionospheric modification in these cases. It is seen from Table 1 that experiment 4 was performed when the heating transmitter power was modulated with $4 \mathrm{~s}$ on every $20 \mathrm{~s}$. The reason for the lack of effects in experiment 4 is probably that the time the heater was on was too short for the nonlinear effects and resultant irregularities to develop, and that X-mode, which usually does not produce strong irregularities, was used for half the satellite pass. Experiment 3 was performed at a time when natural electron density irregularities existed near and within the heated region. In this case it is difficult to distinguish between artificial structures and irregularities caused by natural processes. The record for the satellite pass during experiment 5 contained interference noise from a local source and could not be analysed. Hence in this study only the first two experiments of heating, indicated in Table 1, are discussed.

Table 1. Parameters of the satellite data and HF operation for the different sessions

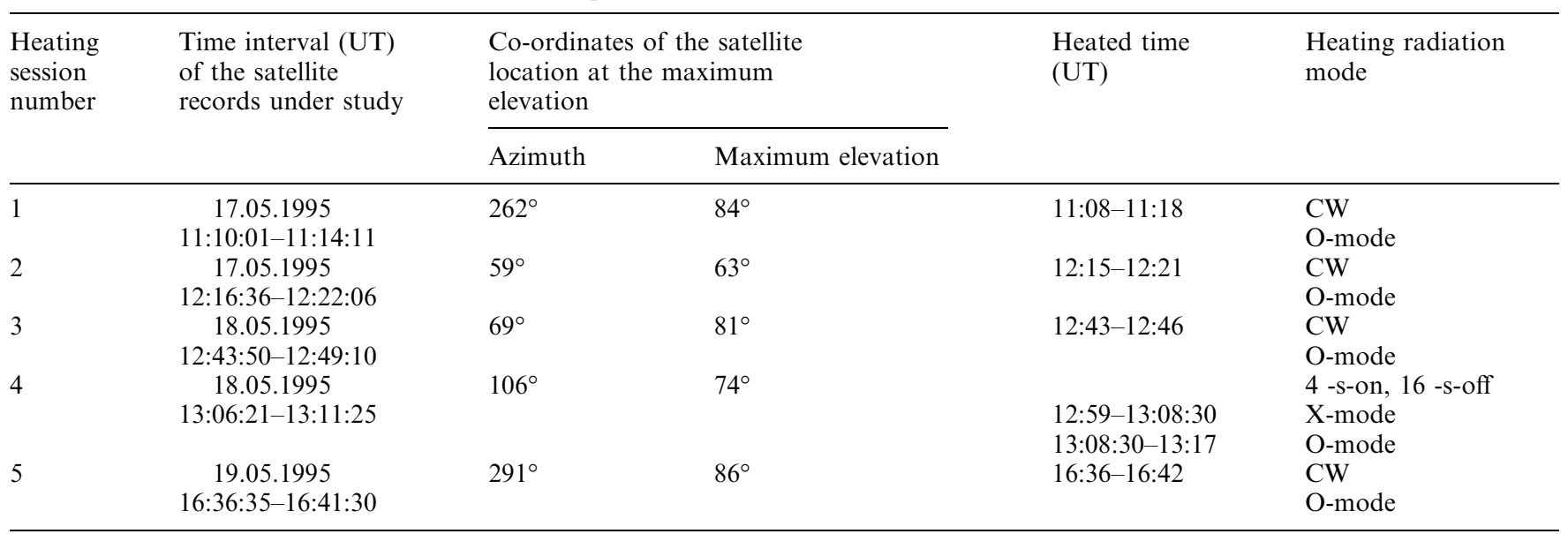




\section{Theoretical background}

An estimation of large-scale electron density structures can be performed by determining the total electron content along satellite-receiver lines. It has been shown by Markkanen et al. (1995) that the total electron content can be obtained from the phase difference, $\varphi$, of the two coherent frequencies (150 and $400 \mathrm{MHz})$ observed on the ground with a constant accuracy as follows:

$\varphi=\left(1-(150 / 400)^{2}\right) \lambda r_{e} \int \mathrm{d} l N+C$

where $\lambda=2 \mathrm{~m}$ is the radio wavelength, $r_{e}=2.82 \times$ $10^{-15} \mathrm{~m}$ is the classical electron radius, $\mathrm{d} l$ is an increment along a satellite-receiver line, $C$ is a constant, $N=N_{0}+\delta N$ is the electron density.

The properties of small-scale irregularities (electron density fluctuations $\delta N$ ) can be investigated by applying a statistical analysis. Since the effects of $\delta N$ on the received signal consist of rapid scintillations, they can be separated from the effects of the average density, $N_{0}$, and studied separately. In this case it is convenient to study, for example, the mean value of the logarithm of the relative amplitude of the field which has a reciprocal relation with the irregularities. We consider the signal observed by a receiver and choose the $z$-axis of the Cartesian co-ordinate system to point from the receiver to the transmitting satellite. The small-scale irregularities are assumed to lie between the satellite and the receiver confined by the region $z_{l}<z<z_{u}$. The signal strength observed on the ground is affected by irregularities scattering at, or close to, the $z$-axis. Its value is statistically determined by the autocorrelation function of the electron density fluctuations integrated over the increment in the $z$-direction. This quantity is defined as

$A_{N}(0, \vec{l})=\int_{-\infty}^{\infty} \mathrm{d}\left(l_{2}-l_{1}\right)\left\langle\delta N\left(\vec{\rho}_{2}, \vec{l}_{2}\right) \delta N\left(\vec{\rho}_{1}, \vec{l}_{1}\right)\right\rangle$

where $\vec{\rho}=(x, y)$ is the vector in the $x y$-plane perpendicular to the direction of wave propagation.

The mean value of the logarithm of the relative amplitude of the signal can be written in the following form

$$
\begin{aligned}
X & =\left\langle\ln \frac{A}{A_{0}}\right\rangle \\
& =-\left.\frac{\lambda^{2} r_{e}^{2}}{4 \pi^{2}} \int_{z_{l}}^{z_{u}} \mathrm{~d} l \int \mathrm{d} \vec{k}_{\rho} \Phi_{N}(\vec{k})\right|_{k_{z}=0} \sin ^{2} \frac{R_{F}(l) k_{\rho}^{2}}{4 \pi},
\end{aligned}
$$

where $A$ is the observed amplitude scattered by the irregularities, $A_{0}$ is the amplitude of the transmitted signal, $k$ is the wave number, $R_{F}(l)=\sqrt{\lambda\left(l_{0}-l\right) l / l_{0}}$ is the radius of the principal Fresnel zone, and $l_{0}$ is the distance between the receiver and satellite. The angular brackets indicate the statistical average.

Expression (2) can be applied to experimental data since $A_{N}(0, \vec{l})$ is defined in terms of the spatial spectral density $\Phi_{N}(\vec{k}, \vec{l})$ as follows:

$$
A_{N}(0, \vec{l})=\left.\frac{1}{(2 \pi)^{2}} \int \mathrm{d} \vec{k}_{\rho} \Phi_{N}(\vec{k}, \vec{l})\right|_{k_{z}=0},
$$

where

$\Phi_{N}(\vec{k}, \vec{l})=\sigma_{N}^{2}(l) \Phi\left(\frac{1}{(2 \pi)^{2}}\left(\left[k_{\perp}^{2}+\alpha^{2} k_{\|}^{2}\right] L_{0}^{2}\right)\right)^{-p / 2}$

where $\sigma_{N}^{2}(l)$ is the variance of the electron density fluctuations, $\Phi$ is the normalised spectral density being a power-law function of a quadratic form, $k_{\perp}$ and $k_{\|}$ indicate the perpendicular to the magnetic field and field aligned wave numbers respectively. Taking into account that the correlation function of the electron density fluctuations is related to the spectral density by a Fourier transform and substituting Eqs. (3) into (2), the expression for $X$ can be written as

$$
\begin{aligned}
X= & -\pi r_{e}^{2}\left(\frac{\lambda}{L_{0}}\right)^{2} \int_{0}^{\infty} \mathrm{d} y \Phi(y) \sin ^{2} y \\
& \cdot \int_{z_{l}}^{z_{u}} \mathrm{~d} l \frac{\sigma_{N}^{2}(l)\left(\sqrt{\pi} \frac{R_{F}(l)}{L_{o}}\right)^{p-2} F\left(1-\frac{p}{2}, \frac{1}{2}, 1, \frac{\gamma(l)}{1+\gamma(l)}\right)}{[1+\gamma(l)]^{1 / 2}}
\end{aligned}
$$

where $\gamma(l)=\left(\alpha^{2}-1\right) \sin ^{2} \Theta(l), p$ is the power law index, $F$ is the hypergeometric function, $\Theta(l)$ is the angle between the direction of the wave propagation and the magnetic field which in general depends on $l$. This equation shows that at high latitudes with the magnetic field lines oriented almost vertically the condition $\Theta(l) \approx 0$ can be applied. That is why the detection threshold for the same field-aligned irregularities is significantly lower at high latitudes than at middle or sub-auroral latitudes.

\section{Experimental results}

Before presenting the results let us consider the geophysical situation during the experiment which has a considerable effect on measurements at high latitudes. On the night preceding May 17, the magnetogramme in Tromsø showed strong magnetic disturbances, associated with high-speed flows from solar coronal holes, until 08 UT with decreasing activity up to 11-12 UT. Therefore, the records for satellite passes above the same region, just before and after the ionosphere modification period, should be seen in order to check the existence of natural irregularities and estimate the artificial ones.

Let us use results (1) and (4) to study the ionospheric condition. Figures 1-4 show the satellite records and modelling results before (Fig. 1), during (Figs. 2 and 3) and after (Fig. 4) the HF heating. They represent the satellite records and modelling results. All these figures use the latitudinal scale of the $x$-axes fixed to an effective ionospheric height of $300 \mathrm{~km}$. The upper panel of the figures presents the measured amplitude of the satellite signal. The next panel below shows the measured phase and its approximation (the smooth curve is the phase 

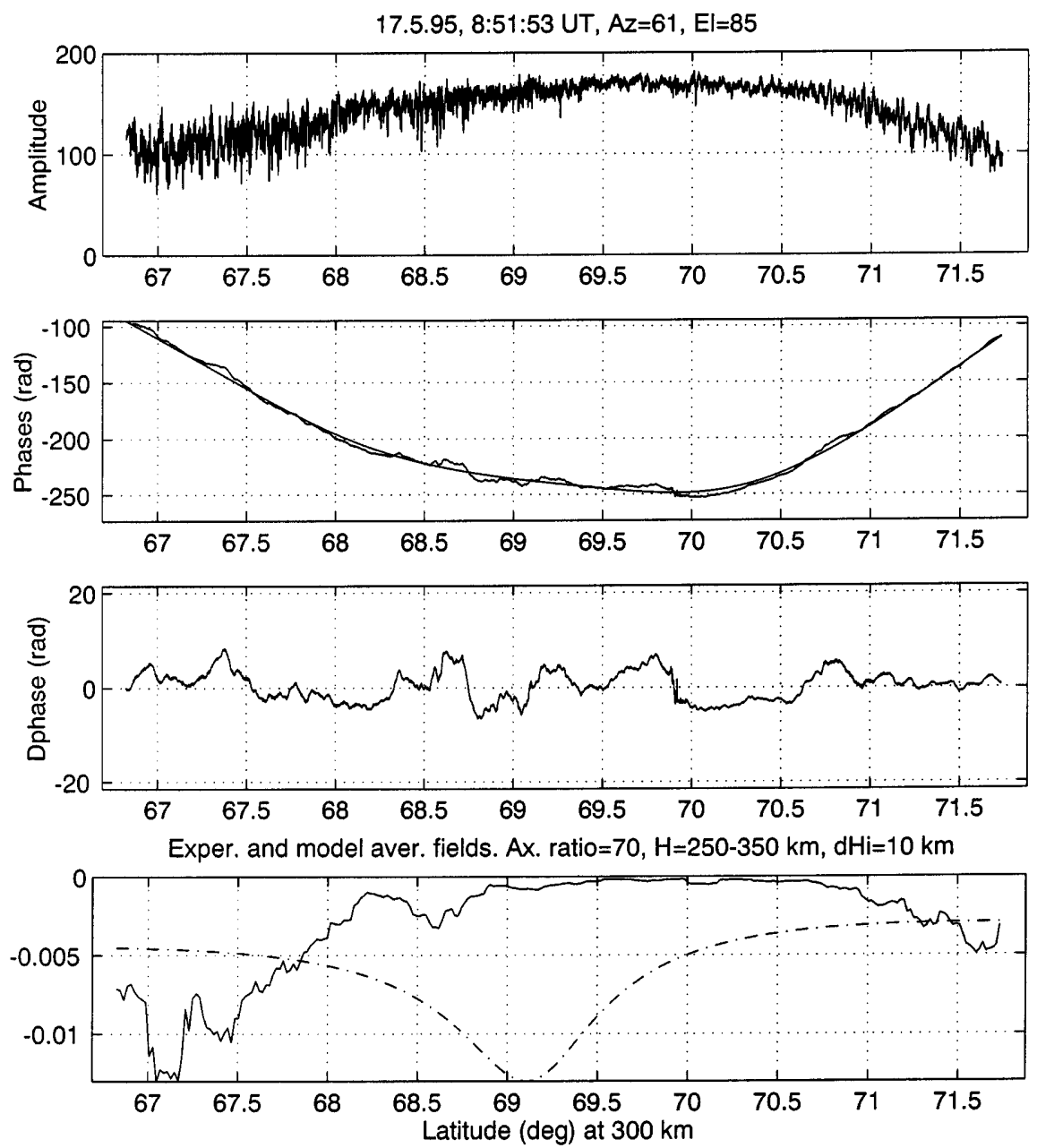

Fig. 1. Experimental and modelling results for the satellite record made about $2 \mathrm{~h}$ before heating. The top panel presents the measured amplitude of the satellite signal. The time at the top indicates the start time of the record, $A z$ and $E l$ are the azimuth and elevation of the satellite's closest approach as seen by the receiver. The second panel from the top shows the measured phase and its approximation (the smooth curve) obtained by lowpass filtering with $0.01 \mathrm{~Hz}$ cut-off. The difference between the measured total electron content and its approximation is shown in the third panel. The solid curve of the 4th panel represents the experimental $X$-value and the dashed curve shows the model of $X$ calculated using a flat ionospheric layer of a constant electron density variance between the heights of $250-350 \mathrm{~km}$ with the axial ratio $\alpha=70$ of the irregularities along and perpendicular to the magnetic field. The value $d H i=10 \mathrm{~km}$ is the integration step. The latitudinal scale of the $x$-axes for all the panels is fixed to the height of $300 \mathrm{~km}$ filtered by a low-pass filter with $0.01 \mathrm{~Hz}$ cut-off). The curve of the third panel noted as dphase is proportional to the difference between the measured total electron content and its approximation. The solid curve of the 4th panel in the figures represents the experimental mean value $X$ of the logarithm of the relative amplitude and the dashed curve shows the model of $X$ which is calculated using Eqs. (3) and (4) and a flat ionospheric layer of a constant electron density variance between $z_{l}=250 \mathrm{~km}$ and $z_{u}=350 \mathrm{~km}$ with the axis ratio $\alpha=70$ of the irregularities along and perpendicular to the magnetic field. The value $d H i=10 \mathrm{~km}$ in the figures is the integration step. The bottom panels of Figs. 2 and 3 also show the spatial cross sections of the calculated antenna pattern of the Tromsø facility as a function $|\sin x / x|$ using the satellite geometry and the parameters of array 2. Free space propagation is assumed here since the effects of refraction are quite small, especially for the upward propagating wave, at the upper-hybrid height which is a few kilometers below reflection and where the production of irregularities is thought to maximise. The open circle on the $x$-axis indicates the latitude of the heating facility.

Figure 1 shows the result of the analysis of the satellite record about $2 \mathrm{~h}$ before the ionospheric modification experiment. It can be seen that the filtered (approximated) phase practically follows a secant law. Therefore, the phase behaviour can be interpreted as a result of the wave propagation through the spherically stratified ionosphere without strong disturbances. After the night-time magnetic storm the ionosphere was not yet in a quiet condition, which is illustrated by the less than 5 radians large-scale variations of the phase. A similar situation can be seen from the amplitude record, where the scintillations take place only in the southern and northern parts of the records. It is more clearly illustrated on the 4th panel from the mean $X$ values of the logarithm of the relative amplitude. In the southern sector between $67^{\circ}-68^{\circ}$ latitudes as well as in the northern part above $71^{\circ}$ latitude we can see the decrease of the average field. An important result from this figure is that small-scale irregularities are not observed in the central zenith part of the passage where we expect the ionosphere to be modified by the powerful radiation. The effect can be noticed at the minimum of the average field at about $69^{\circ}$ predicted by the irregularities model (the dashed curve) which, however, is not seen in the experimental values of $X$ (the solid curve on the same panel). Hence, from Fig. 1 the conclusion can be made that before the heating the ionosphere was weakly disturbed and it contained only weak large-scale electron density variations. 
Figure 2 illustrates the results of the data analysis during the HF-modification. A distinctive difference in these results compared to the previous measurements is a noticeable ionospheric modification above the $\mathrm{HF}$ facility. A displacement of the fluctuation maximum from the direction of the HF-antenna main beam to the sector where the lines-of-sight to the satellite are close to the direction of the magnetic field lines is seen. This fact can be explained as an aspect effect of the ionospheric radio probing when both large- and smallscale irregularities are strongly elongated in the direction of the geomagnetic field. A sharp decrease of the experimental mean value $X$ of the logarithm of the relative field at about $69^{\circ}$ shows that it is difficult to identify the effects of small-scale irregularities away from the field-aligned direction. This is also confirmed by the case illustrated in Fig. 3. Essentially only largescale electron density variations can be observed here, but the influence on the satellite wave of the small-scale irregularities, which could possibly be generated by powerful HF-radiation at latitudes of $69^{\circ}-70^{\circ}$, cannot easily be recognised. For this satellite pass the minimal angle between the magnetic field and the direction to the satellite was very different from zero. That is why in Fig. 3 we do not see a perceptible increase of scintillations which can be ascribed to the heater, although there is a significant level of scintillation which appears to be natural.

The results of the next satellite record (Fig. 4) made about $2 \mathrm{~h}$ after the operation of the HF facility show weaker large-scale fluctuations compared to the data of Figs. 2 and 3, and an enhancement of the background intensity of the mean value of the logarithm of the relative field can also be seen.

Let us estimate the electron density changes at the time of HF ionospheric modification. We start from the large-scale irregularities affecting differential Doppler measurements.
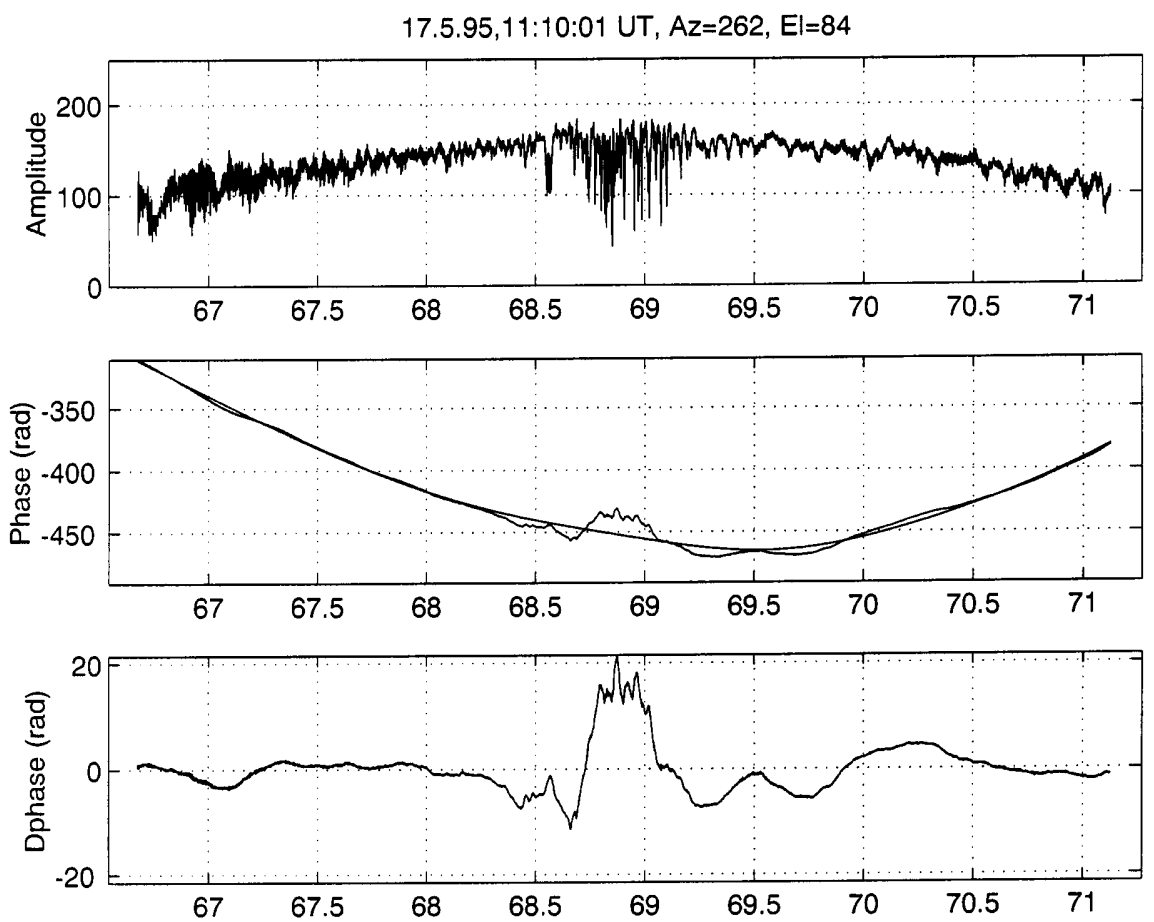

Exper. and model aver. fields. Ax. ratio=70, $\mathrm{H}=250-350 \mathrm{~km}, \mathrm{dHi}=10 \mathrm{~km}$
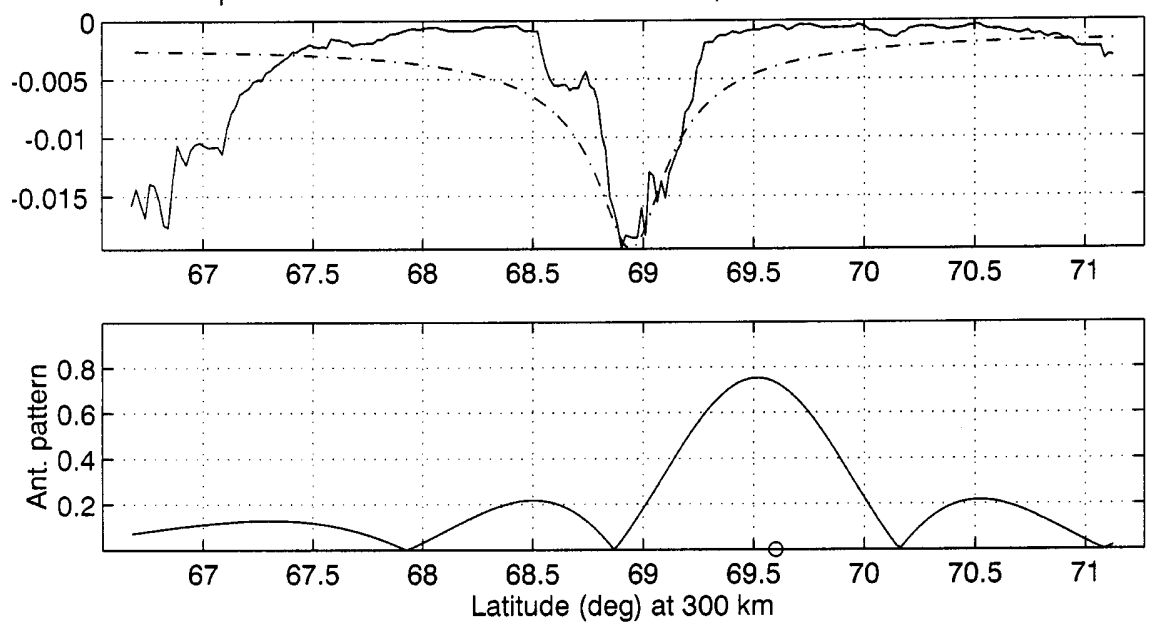

Fig. 2. Same as Fig. 1, but for the satellite pass during the operation of the heating facility. The bottom panel shows the spatial cross section of the calculated antenna pattern of the Tromsø facility as a function $|\sin x / x|$ using the satellite geometry and the parameters of array 2 . The open circle on the $x$-axis indicates the latitude of the heating facility 
E. D. Tereshchenko et al.: Spatial structure of auroral day-time ionospheric electron density irregularities

According to expression (1) we have

$\delta \varphi=\frac{55}{64} \lambda r_{e} \int \mathrm{d} l \delta N_{0}$

where $\delta N_{0}$ is the average density variation. Substituting the numerical values gives

$\delta \varphi=4.85 \cdot 10^{-15} \int \mathrm{d} l \delta N_{0}$.

In order to find the relative changes of the electron density we take into account the fact that the most effective interaction takes place in the region where the plasma frequency is close to $4.04 \mathrm{MHz}$ - the frequency of the radiated wave. We use the well-known formula

$f_{0}^{2}[H z]=80.8 N\left[m^{-3}\right]$.
The electron density corresponding to the plasma frequency of $4.08 \mathrm{MHz}$ measured at the F-region peak obtained from an ionogramme is $2 \times 10^{11} \mathrm{~m}^{-3}$ and we have in our experiment

$\frac{1}{N} \int \mathrm{d} l \delta N_{0}=1.03 \times 10^{3} \delta \varphi$.

From Fig. 3 representing the heating experiment we can find the maximum value $\delta \varphi \approx 15-20$ radians, and the irregularity field elongation can be several tens of kilometers. The ionogramme in Tromsø obtained just before the heating shows a small difference between the maximum frequencies for $F_{1}$ and $F_{2}$-layers and both frequencies being close to the transmitted frequency. Assuming a height extent of $100 \mathrm{~km}$ for the ionosphere we can estimate the relative variation of the electron
17.5.95,12:16:36 UT, $A z=59, E l=63$
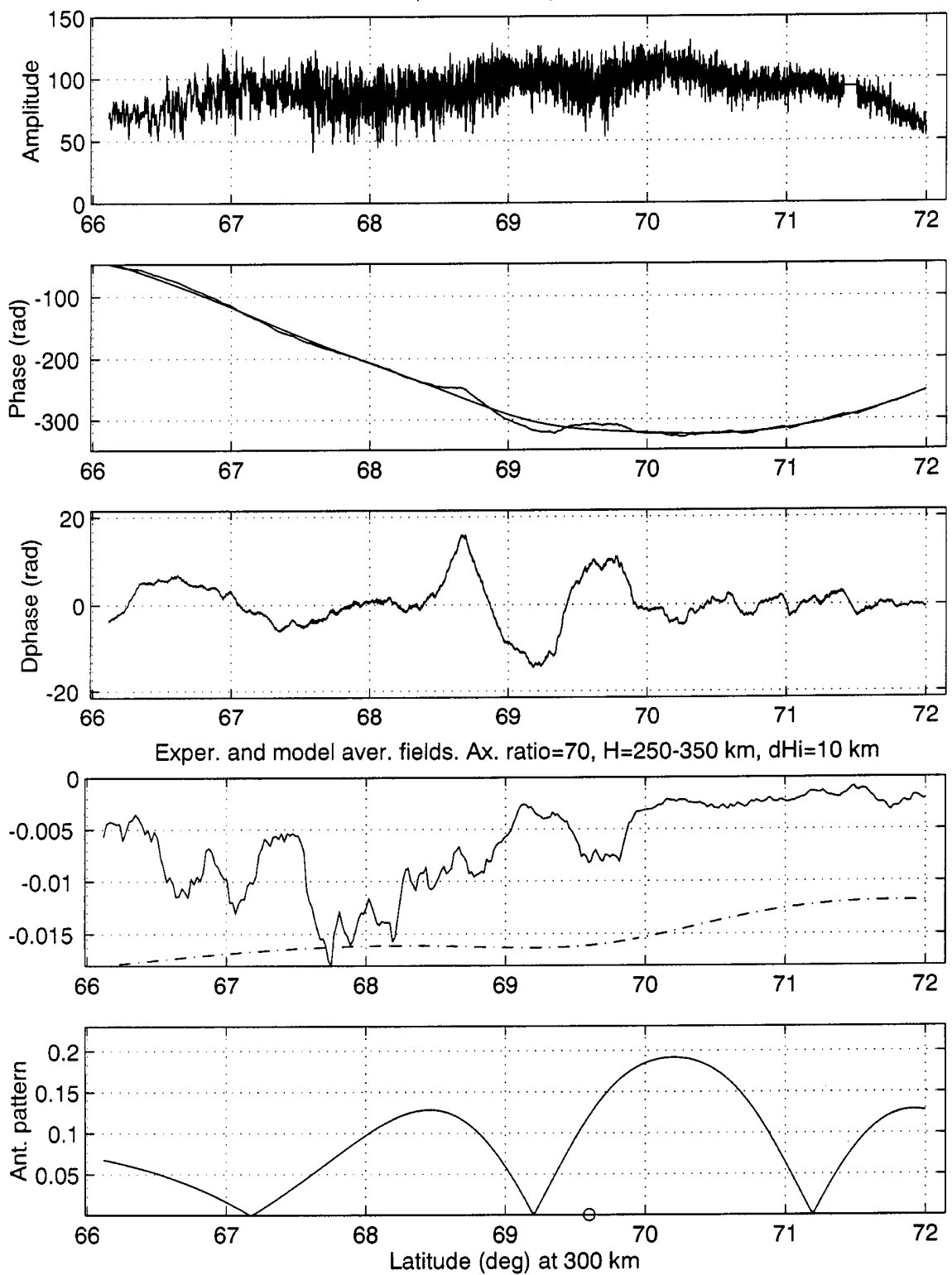

Fig. 3. Same as Fig. 2, but for the next satellite passage during the operation of the heating facility 
density $\delta N_{0} / N_{0} \approx 15-20 \%$ for horizontal scale sizes of $50-100 \mathrm{~km}$.

Next we consider the small-scale irregularities. Note that the majority of models for small-scale electron density inhomogeneities use the parameter $\sqrt{C_{S} L}$, where $L$ is the irregular layer thickness, and the quantity $C_{s}$ has been obtained by Fremouw (1980). In his notation it is expressed as:

$C_{s}=8 \pi^{3 / 2} \frac{\Gamma(p / 2)}{\Gamma((p-3) / 2)} \sigma_{N}^{2}\left(2 \pi / L_{0}\right)^{p-3}$

where $\Gamma$ is the gamma function.

Let us render concrete the function $\Phi$ in expression (3). A commonly used model of a power-law spectrum with a power index $p$ is defined according to the equation

$$
\begin{aligned}
\Phi= & \frac{\left(L_{0} / 2 \pi\right)^{3} \Gamma(p / 2)}{2 \pi \Gamma(3 / 2) \Gamma((p-3) / 2)} \\
& \times\left[\left(\frac{L_{0}}{2 \pi}\right)^{2}\left(k_{\perp}^{2}+\alpha^{2} k_{\|}^{2}\right)\right]^{-p / 2} .
\end{aligned}
$$

Then the mean value $X$ of the logarithm of the relative amplitude for this spectrum has the following form:

$$
\begin{aligned}
X= & -\left(\lambda r_{e}\right)^{2} \frac{\alpha L_{0}}{2 \sin (\pi p / 4) \Gamma((p-3) / 2)} \\
& \times \int_{z_{l}}^{z_{u}} \mathrm{~d} z \frac{\sigma_{N}^{2}(l)\left(\sqrt{\pi} \frac{R_{F}(l)}{L_{o}}\right)^{p-2} F\left(1-\frac{p}{2}, \frac{1}{2}, 1, \frac{\gamma(l)}{1+\gamma(l)}\right)}{[1+\gamma(l)]^{1 / 2}} .
\end{aligned}
$$

It has been shown by Kunitsyn and Tereshchenko (1991) that for the ionospheric radiowave probing the relation between the measured value $X_{\exp }$ of the logarithm of the relative amplitude and its model value $X$ can be written as

$X_{\exp }=X / 2$.

Having assumed the electron density variance being constant within the irregular region we can estimate the level of $\sqrt{C_{S} L}$ corresponding to the results obtained in the HF heating experiment. We use the expression

$$
\begin{aligned}
\sqrt{C_{S} L}= & 4 \sqrt{\frac{\alpha \Gamma(p / 2) \sin \frac{p \pi}{4} \cos \Theta_{n}\left(-X_{\exp }\right)}{\lambda r_{e}}} \\
& \times\left(\frac{R_{F}}{2 \sqrt{\pi}}\right)^{1-p / 2}
\end{aligned}
$$

17.5.95, 14:01:12 UT, $A z=265, E l=57$
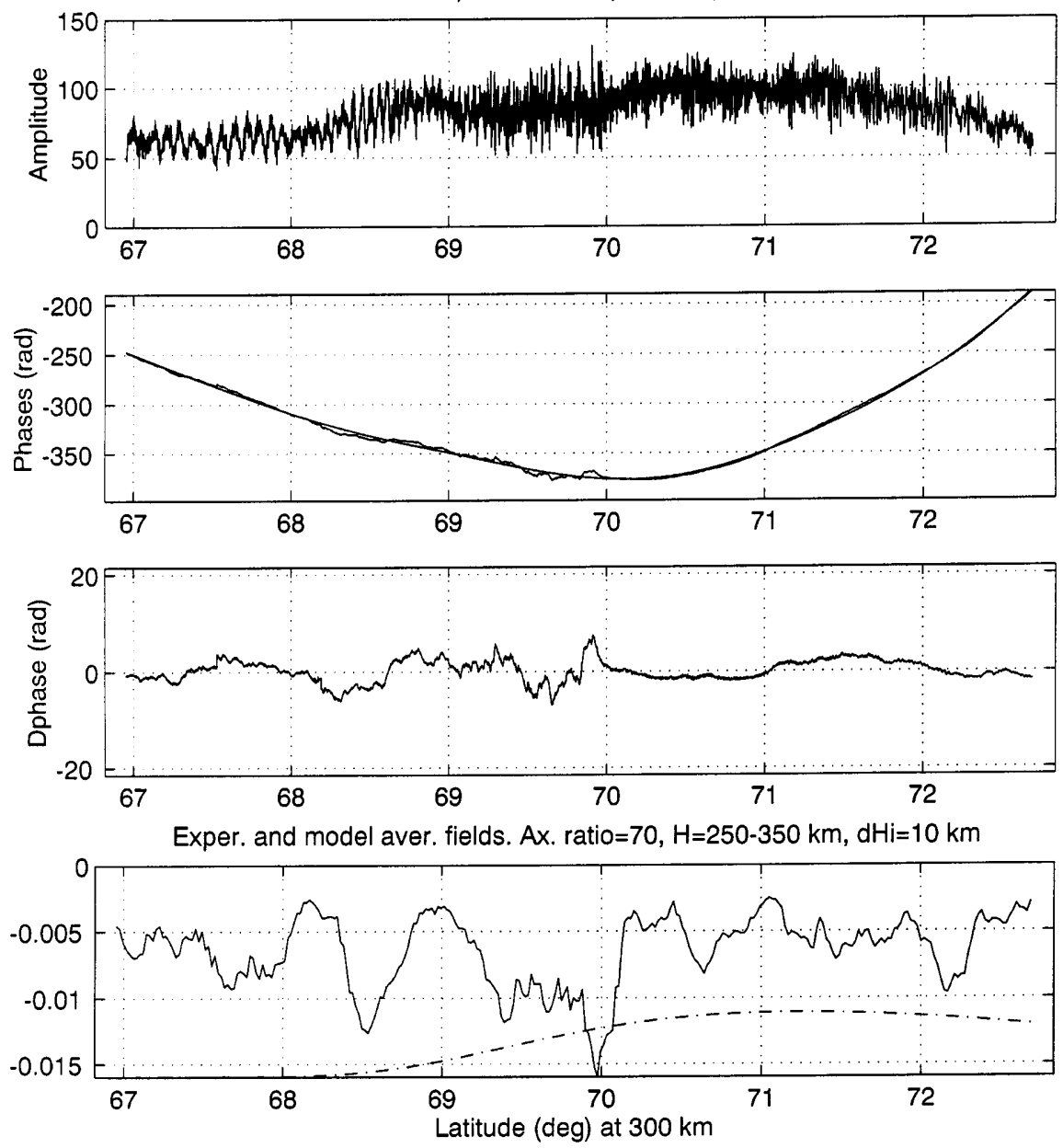

Fig. 4. Same as Fig. 1, but for the satellite pass about $2 \mathrm{~h}$ after the heating experiment presented in Fig. 3 
where $\Theta_{n}$ is the angle between the line-of-sight to the satellite and the normal to the spherical irregular layer. In our case under study it is about $13^{\circ}$. The power index in expression (12) for $\sqrt{C_{s} L}$ can be easily obtained from the experimental data.

Figure 5 shows the power spectral densities (PSD) obtained for the amplitude de-trended over a 10-s interval, and for small-scale variations of the phase filtered by a low-pass filter with $0.08 \mathrm{~Hz}$ cut-off. The straight lines on the spectra are the fitted lines, and the spectral power indices $p \approx 3.8$. Inserting the value $p=3.8$ into the formula for $\sqrt{C_{s} L}$ produces $\sqrt{C_{s} L} \approx$ $4.3 \times 10^{11}$. It can be noted that this value is in good agreement with scintillation modelling results obtained by Fremouw et al. (1985) for natural irregularities. Sometimes applying the spectrum definition, the parameter $C_{N}$ is employed using the relationship $C_{N}=\alpha C_{s} / 8 \pi^{3}$ and the value $\sqrt{C_{N} L} \approx 2 \times 10^{10}$ is obtained.

\section{Conclusion}

We have described the results of satellite radio probing of the high-latitude ionosphere modified by the powerful radiation of the HF heating facility at Tromsø resulting in the generation of both large-scale irregularities (several tens of kilometers in size) and small-scale ones (from hundreds of meters to kilometers). It is shown that the maximum irregularity development in satellite signals follows the direction parallel to the geomagnetic field lines. The intensity of small-scale irregularities produced is not so high. However a strong elongation of the irregularities in the direction of the magnetic field makes it possible to observe their features in the corresponding sector using the aspect-angle effect. That is why a displacement of the fluctuation maximum from the heated region is seen to the south where the line-ofsight to the satellite is close to the direction of the magnetic field line. Large-scale structures of the electron density can be detected within a much larger region.

An important result obtained from expression (10) is that amplitude measurements in a given direction contain information about the integral of the variance of the electron density fluctuations, over a line from the satellite to the receiver. Therefore, analogous to the usual ray tomography where phase shift measurements are proportional to the total electron content along the ray, amplitude measurements of the mean value of the logarithm of the relative amplitude of the signal on a chain of receivers give the integrals of the electron density variance along a set of lines crossing each other in the ionosphere and a 2-dimensional distribution of the variance can be determined by a new method of amplitude tomography providing ionospheric electron
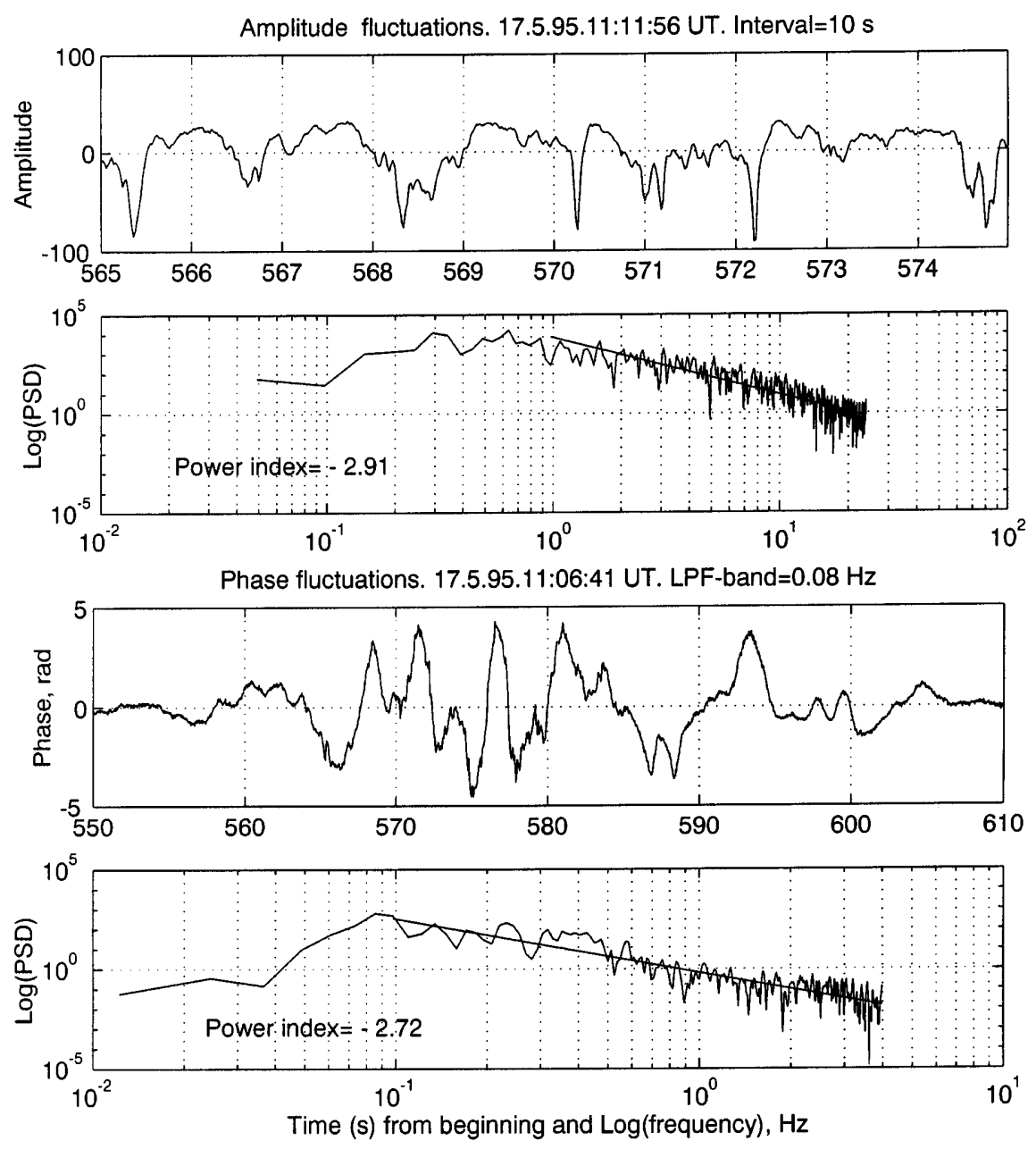

Fig. 5. Amplitude and phase fluctuations of the satellite signal and their power spectral densities (PSD) for the experiment shown in Fig. 2. The PSD of the amplitude (second panel from the top) is obtained from the detrended amplitude in a 10 s-interval (top panel). The PSD of the phase (bottom panel) is computed from the small-scale variations of the phase filtered by a low-pass filter with $0.08 \mathrm{~Hz}$ cut-off (third panel). The $x$-axes of the $2 n d$ and $3 r d$ panels show seconds from the data acquisition start time at 11:02:31 UT 
density maps and a detailed investigation of properties of natural and artificially produced irregularities.

Though the experiment was executed at the EISCAT site we, unfortunately, had no incoherent scatter data which could allow us to obtain more informative geophysical data by using extra measurements of the electron density distribution, electron temperature, etc. as well. This short experiment can be considered as a necessary initial step for future measurements by the radio tomographic technique to investigate the distribution of the ionospheric irregularities artificially produced by a powerful HF wave.

Acknowledgements. The support of the EISCAT Scientific Association, making it possible to perform the experiment, is gratefully acknowledged. EISCAT is an International Association supported by Finland (SA), France (CNRS), the Federal Republic of Germany (MPG), Japan (NIPR), Norway (NFR), Sweden (NFR) and the United Kingdom (PPARC). The authors wish to thank O. Evstafjev from the Polar Geophysical Institute for his help in preparing computer programs.

Topical Editor D. Alcaydé thanks S. Basu and E. Honary for their help in evaluating this paper.

\section{References}

Basu S., S. Basu, P. Stubbe, H. Kopka, and J. Waaramaa, Daytime scintillations induced by powerful HF waves at Tromsø, Norway, J. Geophys. Res. 92, 11, 149-157, 1987.
Costa, E., S. Basu, R. C. Livingston, and P. Stubbe, Multiple baseline measurements of ionospheric scintillation induced by high-power HF waves, Radio Sci., 32, 1, 191-197, 1997.

Farley D. T., Artificial heating of the electrons in the F region of the ionosphere, J. Geophys. Res., 68, 401-410, 1963.

Fremouw E. J., Geometrical control of the ratio of intensity and phase scintillation indices, J. Atmos. Terr. Phys., 42, 775-787, 1980.

Fremouw E. J., J. A. Secan, and J. M. Lansinger, Spectral behaviour of phase scintillation in the nighttime auroral region, Radio Sci., 20, 923-933, 1985.

Ginsburg V. L., and A. V. Gurevich, Nonlinear phenomena in a plasma located in an alternating electric field. Sov. Phys. Usp. (English Transl.) 3, 115, 1960.

Gurevich A. V., Nonlinear phenomena in the ionosphere, SpringerVerlag, New York Berlin Heidelberg 1978.

Kunitsyn V. E., and E. D. Tereshchenko, Radiotomography of the ionosphere. Moscow, Nauka, 1991 (in Russian).

Markkanen, M., M. Lehtinen, T. Nygrén, J. Pirttilä, P. Henelius, E. Venelius, E. D. Tereshchenko, and B. Z. Khudukon, Bayesian approach to satellite radio tomography with applications in the Scandinavian sector, Ann. Geophysicae, 13, 1277-1287, 1995.

Perkins F. W. and E. J. Valeo, Thermal self-focusing of electromagnetic waves in plasmas, Phys. Rev. Lett., 32, 1234-1237, 1974.

Rietveld M. T., H. Kohl, H. Kopka, and P. Stubbe, Introduction to ionospheric heating at Tromsø - I. Experimental overview, J. Atmos. Terr. Phys., 55, 577-599, 1993.

Stubbe P., Review of ionospheric modification experiments at Tromsø, J. Atmos. Terr. Phys., 58, 349-368, 1996.

Wood C. D., and G. E. Perry, The Russian satellite navigation system, Philos. Trans. R. Soc., London, A, 294, 307-315, 1980. 